\title{
Secretin induces neurite outgrowth of PC12 through cAMP-mitogen-activated protein kinase pathway
}

\author{
Hyeon Soo Kim ${ }^{1}$, Sanatombi Yumkham ${ }^{1}$, \\ Sun-Hee Kim ${ }^{1}$, Kyungmoo Yea ${ }^{1}$, \\ You Chan Shin ${ }^{1}$, Sung Ho Ryu ${ }^{1}$ \\ and Pann-Ghill Suh ${ }^{1,2}$ \\ ${ }^{1}$ Department of Life Science \\ Division of Molecular and Life Science \\ Pohang University of Science and Technology \\ Pohang 790-784, Korea \\ ${ }^{2}$ Corresponding author: Tel, 82-54-279-2293; \\ Fax, 82-54-279-0645; E-mail, pgs@postech.ac.kr
}

Accepted 1 February 2006

Abbreviations: MAPK, mitogen-activated protein kinase; NGF, nerve growth factor; PKA, protein kinase A; VIP, vasoactive intestinal peptide

\begin{abstract}
The gastrointestinal functions of secretin have been fairly well established. However, its function and mode of action within the nervous system remain largely unclear. To gain insight into this area, we have attempted to determine the effects of secretin on neuronal differentiation. Here, we report that secretin induces the generation of neurite outgrowth in pheochromocytoma PC12 cells. The expressions of Tau and beta-tubulin, neuronal differentiation markers, are increased upon secretin stimulation. In addition, secretin induces sustained mitogen-activated protein kinase (MAPK) activation and also stimulates the CAMP secretion. Moreover, the neurite outgrowth elicited by secretin is suppressed to a marked degree in the presence of either PD98059, a specific MAPK/ERK kinase (MEK) inhibitor, or H89, a specific protein kinase $A$ (PKA) inhibitor. Taken together, these observations demonstrate that secretin induces neurite outgrowth of PC12 cells through cAMP- MAPK pathway, and provide a novel insight into the manner in which secretin participates in neuritogenesis.
\end{abstract}

Keywords: mitogen-activated protein kinases; neurites; pheochromocytoma; secretin; tau proteins

\section{Introduction}

Secretin has been shown to belong to a peptide family which includes vasoactive intestinal polypeptide (VIP), pituitary adenylate cyclase-activating polypeptide (PACAP), and glucagon-like peptide. Members of this peptide family have also been determined to function as ligands for known Gprotein-coupled receptors (Dong and Miller, 2002). Most of the peptides in this group have been detected both peripherally and centrally, thereby reflecting their known hormonal role within the gastrointestinal system, and suggesting that they may also play a role in neuronal function. Furthermore, secretin receptors have been shown to be expressed in mammalian brain tissues, including the cerebellum (Fremeau et al., 1983; Nozaki et al., 2002).

Rat pheochromocytoma PC12 cells have been previously utilized in a model system for the study of the intracellular signaling pathway which leads to neuronal differentiation. Stimulation with differentiating factors, including nerve growth factor (NGF), and the subsequent activation of its receptor, TrkA, were demonstrated to initiate a variety of signaling events which led to the cessation of growth, the acquirement of electrical excitability, the expression of neuron-specific marker proteins, and the extension of neurites (Hagag et al., 1986; Leoni et al., 1999; Grumolato et al., 2003). Neurite extension induced by different stimuli was determined to be a distinctive aspect of neuronal differentiation (Bouron et al., 1999).

The signal pathways involved in neuronal differentiation have been studied extensively. Sustained mitogen activated protein kinase (MAPK) activation has been confidently implicated in NGF-induced neurite outgrowth occurring in PC12 cells (Traverse et al., 1992; Nguyen et al., 1993; Pang et al., 1995). Neurite outgrowth in PC12 cells has been experimentally induced by NGF, but not by epidermal growth factor (EGF). This differential response was attributed to the difference in the duration of MAPK activation; NGF induced a sustained MAPK activation, but EGF resulted in only a short-lived activation (Yasui et al., 2001). On the other hand, the regulation of intracellular cAMP levels has been implicated in the control of a variety of biological functions, including neuronal differentiation. cAMP appears to generate a powerful survival and differen- 
tiation signal for neurons (Rydel and Greene, 1988; Piiper et al., 2002). Under serum-free conditions, CAMP was shown to sustain survival, and to promote the neurite outgrowth in neurons. cAMPinduced neuronal differentiation has been generally thought to be involved in the phosphorylation of transcription factors (Montminy, 1997). However, the identity of the intracellular signaling molecules which mediate neuronal differentiation has yet to be precisely determined.

The objective of this study was to obtain more insight into the molecular mechanisms involved in secretin-mediated neuritogenesis. In this study, we have evaluated the effects of secretin on both MAPK activity and CAMP generation. Here, we present the data suggesting that the cAMP-MAPK pathway might be involved in secretin-mediated neuritogenesis.

\section{Materials and Methods}

\section{Reagents}

The synthetic secretin and VIP used in this study were obtained (Phoenix Pharmaceuticals, Inc. Mountain View, CA). The anti-phosphoTrkA/Akt/ERK1 /2(Thr 202/Thy 204), anti-Akt/ERK1/2, and anti-beta tubulin antibodies were purchased (New England Biolabs, Beverly, MA) and the secondary antibodies conjugated to horse radish peroxidase were bought (Kirkegaard and Perry Lab, Gaithersburg, MD). The NGF was purchased (Upstate Biotechnology Incorporated, Lake Placid, NY). The dibutyryl-cAMP, H89, and rapamycin were bought (Calbiochem, Strasbourg, France). The PD98059 was obtained (Alexis, Coger, France).

\section{Cell culture}

The PC12 cells were grown in culture medium containing $500 \mathrm{ml}$ of RPMI 1640 (GIBCO $^{\mathrm{TM}}$, Auckland, NZ) supplemented with $0.584 \mathrm{~g} /$ liter L-glutamate and $4.5 \mathrm{~g} /$ /iter of glucose, mixed with $500 \mathrm{ml}$ of $\mathrm{F}-12$ medium containing $0.146 \mathrm{~g} /$ liter of L-glutamate, $1.8 \mathrm{~g} /$ liter of glucose, $100 \mu \mathrm{g} / \mathrm{ml}$ of gentamicin, 2.5 $\mathrm{g} /$ liter of sodium carbonate, $5 \%$ heat-inactivated fetal bovine serum (FBS), and $10 \%$ horse serum.

\section{Immunoblot analysis}

The PC12 cells were allowed to grow in 6-well plates until achieving 60-70\% confluence, after which they were serum-starved for 24 hours prior to the administration of treatment with selected agents. The media were then aspirated, and the cells were washed twice in ice-cold PBS, then lysed in $100 \mu$ of lysis buffer $(0.5 \%$ deoxycholate, $0.1 \%$ SDS, $1 \%$
Nonidet P-40, $150 \mathrm{mM} \mathrm{NaCl}$ and $50 \mathrm{mM}$ Tris- $\mathrm{HCl}$, $\mathrm{pH}$ 8) supplemented with proteinase inhibitors $(0.5$ $\mu \mathrm{M}$ aprotinin, $1 \mu \mathrm{M}$ phenylmethylsulfonyl fluoride, 1 $\mu \mathrm{M}$ leupeptin) (Sigma-Aldrich, St. Louis, MO). These samples were sonicated briefly, heated for 5 minutes at $95^{\circ} \mathrm{C}$, then centrifuged for another 5 minutes. The supernatants were electrophoresed on SDS-PAGE $(8-16 \%)$ gradient gels, and transferred to polyvinylidene difluoride membranes. The blots were then incubated overnight at $4^{\circ} \mathrm{C}$ with primary antibodies, and washed 6 times with Tris-buffered saline/0.1\% Tween 20 prior to 1 hour of probing with horseradish peroxidase-conjugated secondary antibodies at room temperature. The blots were then visualized using ECL (Amersham Biosciences, Buckinghamshire, UK). In some cases, the blots were stripped and reprobed with other antibodies.

\section{Immunocytochemistry}

The PC12 cells were plated on poly-L-lysine-coated glass coverslips and maintained in growth media. After 1 day of culture, this medium was replaced with media containing $1 \%$ FBS supplemented with secretin or NGF, then cultured for an additional 2 days. For the immunofluorescence microscopy, the cultured cells were washed in PBS and fixed in 4\% paraformaldehyde in PBS for 1 hour, then permeabilized for 15 minutes with $0.1 \%$ Triton X-100 and blocked with $20 \%$ skimmed milk for 30 minutes. The cells were incubated for 1 hour with primary anti-Tau antibody. After another washing with PBS, the cells were incubated with secondary FITCconjugated monoclonal antibody (Molecular probes, Eugene, OR) for 1 hour. After incubation, the samples were washed in PBS, and then visualized using a confocal microscope (Zeiss LSM 510 Meta, Oberkochen, Germany).

\section{Neurite outgrowth}

The PC12 cells were plated on six-well collagencoated plates in growth media. After 1 day, the medium was changed to $1 \%$ FBS medium containing $1 \mu \mathrm{M}$ of secretin, and incubated for an additional 2 days. Then, the numbers and lengths of neurite outgrowth were counted and measured. Cells with neurite lengths of at least twice the diameter of the cell body were considered to be differentiated cells. All images were captured with a confocal microscope using the $\times 20$ objective (Zeiss LSM 510 Meta, oberkochen, Germany).

\section{RT-PCR}

Levels of secretin receptor and GAPDH expression were determined via RT-PCR, as was described 
A

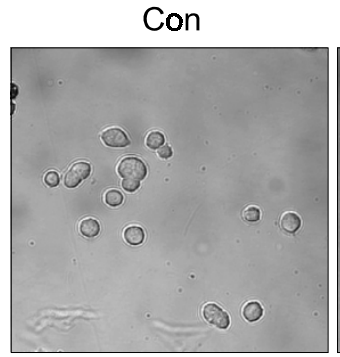

B

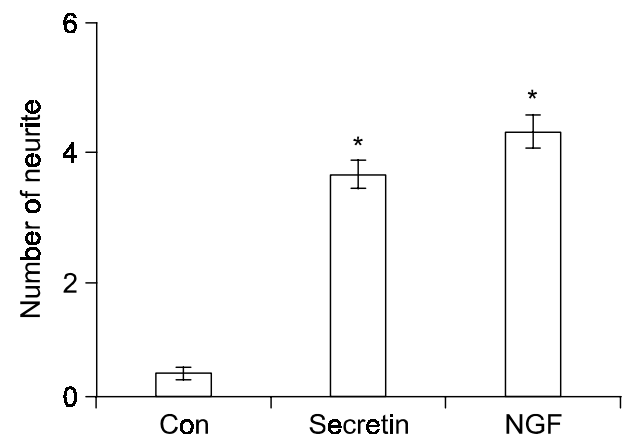

C

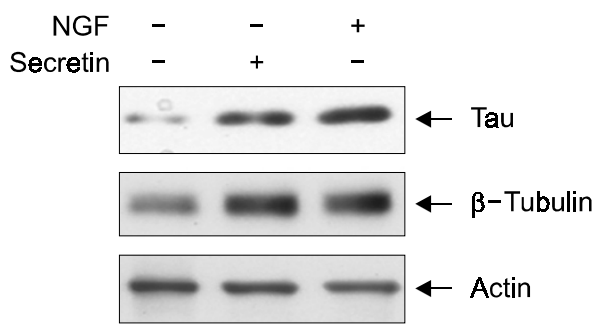

D

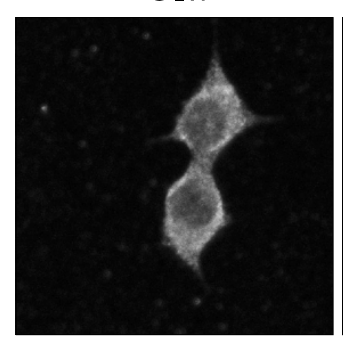

Secretin
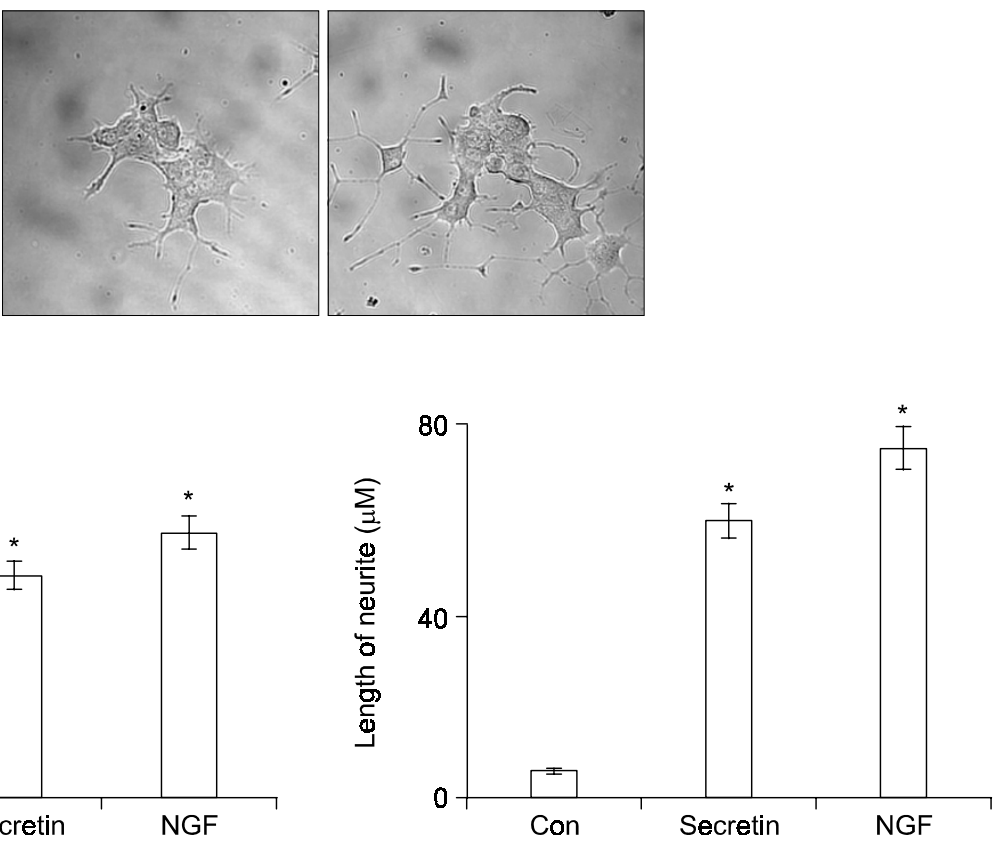

E

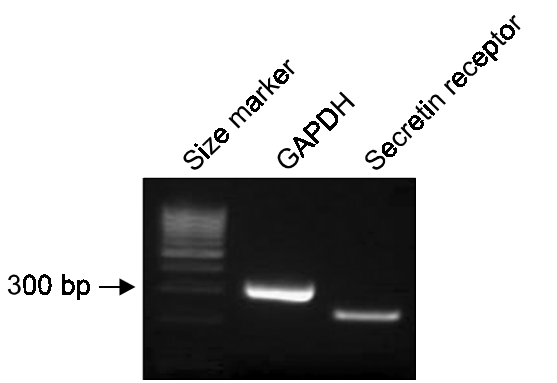

NGF
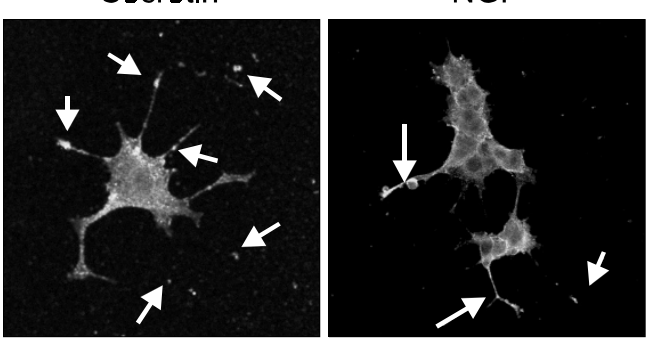

Figure 1. Secretin induces the neurite outgrowth in PC 12 cells. PC12 cells were cultured in RPMI 1640 medium supplemented with $10 \%$ horse serum and $5 \%$ fetal bovine serum. A, in comparison to the control, both secretin and NGF induced the outgrowth of neurites from the cell body. The cells were incubated for 2 days in 1\% FBS medium supplemented with secretin $(1 \mu \mathrm{M})$ secretin and NGF $(10 \mathrm{ng} / \mathrm{ml})$. Phase-contrast photographs were obtained using a confocal microscope, with the $\times 20$ objective. $B$, the percentage of neurite-containing cells was calculated from 5 random fields in one well. The data are expressed as a mean percentage of 4 wells in one representative experiment. Statistical analyses were performed. The data are expressed as means \pm S.E. ${ }^{*} P<0.01$ compared with untreated cells. C, effect of secretin on Tau or beta-tubulin expression. The cells were incubated for 2 days in 1\% FBS medium supplemented with secretin and NGF. The cell lysates were analyzed via immunoblotting with anti-Tau antibody. Protein loading was normalized via immunoblotting with anti-actin antibody. D, the PC12 cells were plated on collagen-coated cover glasses. After 24 hours, the PC12 cells were stimulated for 2 hours with either secretin or NGF. After incubation with primary anti-Tau antibody and secondary FITC-conjugated monoclonal antibody, we captured the images with a confocal microscope using the $x 20$ objective. E, RT-PCR analysis of secretin receptors in PC12 cells. Total RNA was prepared from the PC12 cells and RT-PCR was conducted using a specific secretin receptor primer. The PCR products were then gel-run in $2 \%$ agarose, and visualized in UV. GAPDH mRNA was employed as a positive control. 
below. The synthetic oligonucleotide primer sequences for secretin receptor and GAPDH were as follows; GAPDH(forward), 5'-CGATGCTGGCGCTGAGTAC-3', GAPDH(reverse), 5'-CGTTCAGCTCAGGGATGACC-3' and secretin receptor (forward), 5'TGTTCGTGTCCTTCATCCTG-3', secretin receptor (backward), 5'-GAAGGAGATGGCAAGGAGTG-3'. We conducted PCR amplification on the above-listed primers in a $25-\mu$ l reaction tube. The following program was utilized for 30 cycles of amplification in a Gene Amp PCR System 2400 (Perkin Elmer, Wellesley, MA): 30 seconds at $94^{\circ} \mathrm{C}, 30$ seconds at $62^{\circ} \mathrm{C}$ and 30 seconds at $72^{\circ} \mathrm{C}$. The amplified DNA fragments were then electrophoresed on $2 \%$ agarose gel and, and stained with ethidium bromide.

\section{cAMP measurement}

The cAMP content in the PC12 cell lysates were determined via commercial ELISA assays (Amersham Biosciences, Piscataway, NJ). The cells were preincubated with $1 \mathrm{mM}$ IBMX for 15 minutes, in order to inhibit any endogenous cAMP-phosphodiesterase. The cells were then treated for 10 minutes with the specified agents. The cAMP contents were calculated using a standard curve, and normalized to total amylase contents.

\section{Data analysis}

Data are expressed as the means \pm S.E.M. Statistical analyses were conducted using SigmaStat (SPSS Inc., Chicago). Differences were regarded as significant at a $p$ value of $<0.05$. Comparisons between basal and stimulated status were analyzed via ANOVA with Holm-Sidak comparisons.

\section{Results}

\section{Secretin induces the neurite outgrowth in PC 12 cells}

To address secretin's functions in neuronal differentiation, we first investigated the effects of secretin on the neurite outgrowth of pheochromocytoma PC12 cells. The application of secretin (1 $\mu \mathrm{M})$ was found to induce the generation of neurite outgrowth in these cells (Figure 1A). Both the number of neurites and the length of the branches were determined to have increased as the result of secretin stimulation (Figure 1B). NGF, a well-known differentiating factor, was employed in an effort to induce the neurite outgrowth, and was used as a positive control. Tau, a microtubule-associated protein which is also used as a neuronal differential marker, was found to be unique neuronal component which stabilized microtubules, resulting in the formation of axonal processes (Caceres and Kosik, 1990; Esmaeli-Azad et al., 1994; Hirokawa et al., 1996). To ascertain whether secretin-induced neurite outgrowth was correlated with the induction of the protein, we conducted Western blot analyses to determine the expressions of neuronal differentiation markers, Tau and beta-tubulin. The levels of these proteins were increased by secretin, and were also increased as the result of NGF treatment $(10 \mathrm{ng} / \mathrm{mL})$ (Figure 1C). Our cytochemical analyses showed that the subcellular localization of Tau was enriched in the neurites, especially in the branch regions, after stimulation with secretin (Figure 1D). Next, in order to confirm the existence of the secretin receptor in the PC12 cells, we conducted an RT-PCR experiment. Secretin receptor mRNA was clearly shown to be expressed in these cells (Figure 1E). GAPDH mRNA was constitutively expressed, and was used as a positive control for the RT-PCR experiment. These results clearly showed that secretin possessed the ability to induce the neurite outgrowth in the PC 12 cells.

\section{The MAPK pathway is involved in the secretin-induced neuritogenesis}

To better understand the signal pathways involved in neuritogenesis, we attempted to assess the effects of secretin on the activity of several signal pathways. The phosphorylation of p42/44 MAPK was increased by secretin $(1 \mu \mathrm{M})$ as well as NGF $(10 \mathrm{ng} / \mathrm{ml})$ (Figure $2 \mathrm{~A}, \mathrm{~B})$. The phosphorylation patterns in these cells reached maximal levels at 10 minutes, and persisted for 3 hours. These results were consistent with previous reports that sustained MAPK activation was crucial for neuritogenesis in PC12 cells (Yasui et al., 2001). To confirm the involvement of the MAPK pathway, we pre-treated the cells with PD98059 (2 $\mu \mathrm{M})$ prior to secretin treatment, and then assessed the degree of differentiation in the cells. The neurite outgrowth induced by both secretin and NGF was suppressed significantly by PD98059 pretreatment (Figure 2C). Rapamycin $(10 \mu \mathrm{M})$ was used as a negative control and showed no effects on secretin or NGF-induced neurite outgrowth. The stimulatory effects of these two components on MAPK disappeared in the presence of PD98059 (Figure 2E). These results suggested that the MAPK pathway might be associated with secretin-induced neuritogenesis.

\section{Secretin stimulates cAMP generation in PC12 cells}

To elucidate the molecular mechanisms underlying secretin-induced neuronal differentiation, we then attempted to characterize the effects of secretin on 
A

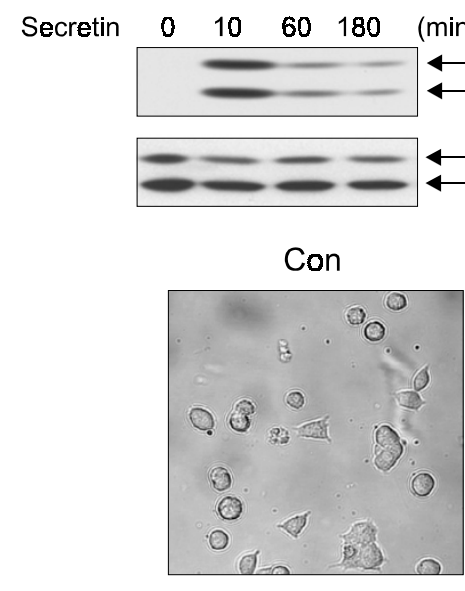

B

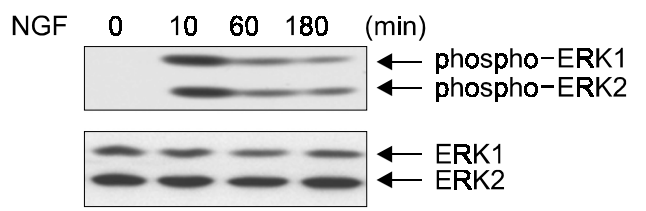

Secretin

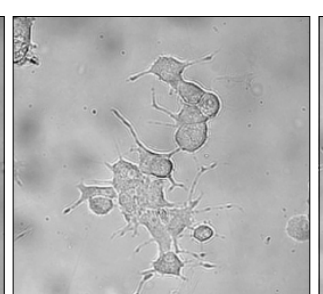

NGF

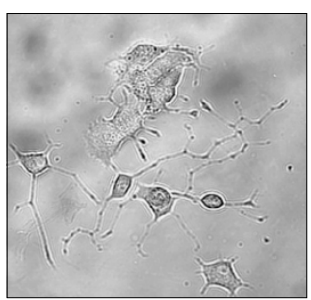

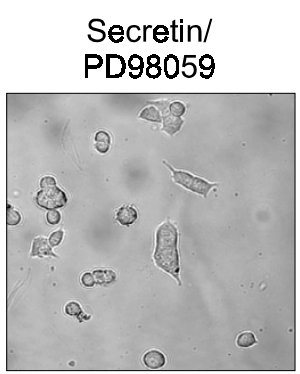

NGF/

PD98059

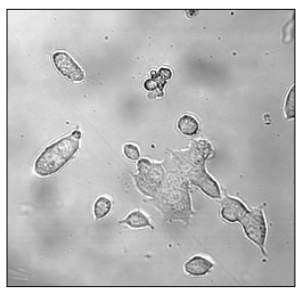

Rapamycin

NGF/

Rapamycin
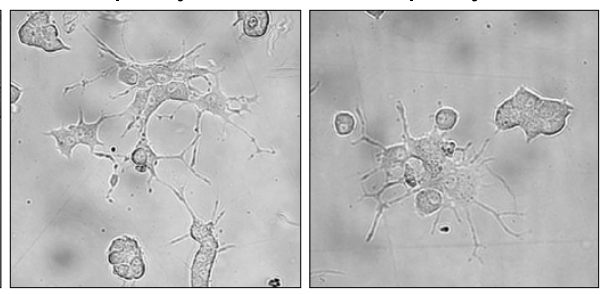

D
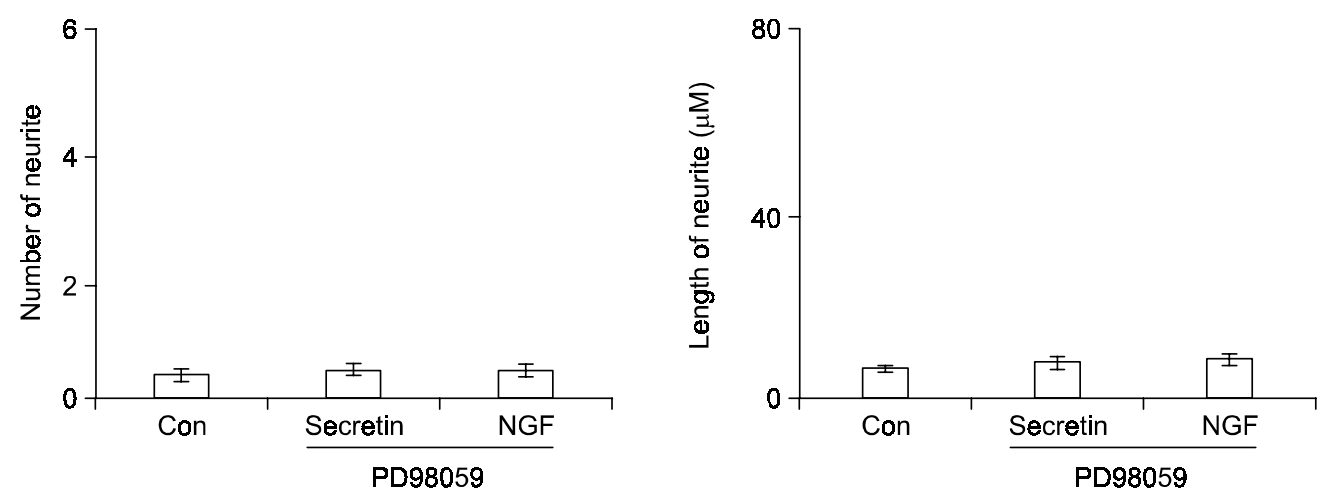

E

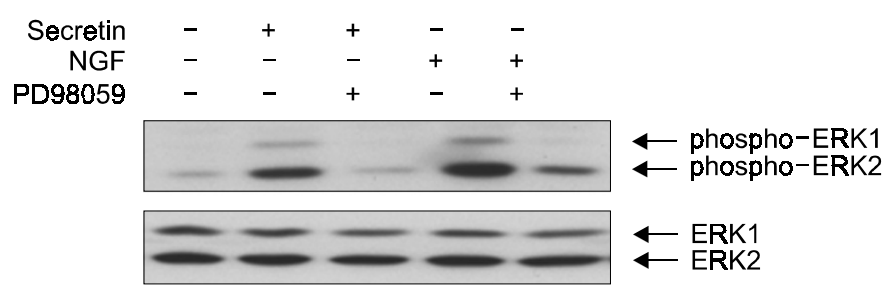

Figure 2. The MAPK pathway is involved in the secretin-induced neuritogenesis. $A$ and $B$, the effects of secretin and NGF on MAPK activity. PC12 cells were stimulated with either secretin $(1 \mu \mathrm{M})$ or NGF $(10 \mathrm{ng} / \mathrm{ml})$ for the indicated times. The cell lysates were then analyzed via immunoblotting with anti-phospho ERK1/2 antibodies. Protein loading was normalized via immunoblotting with anti-ERK1/2 antibodies. C, the effects of PD98059 on neurite outgrowth. PC12 cells were plated on collagen-coated dishes. After 24 hours, the cells were incubated in $1 \%$ FBS medium supplemented with either secretin or NGF, in the presence of PD98059 $(2 \mu \mathrm{M})$ or rapamycin $(10 \mu \mathrm{M})$, respectively. After 2 days, the cells were photographed with a confocal microscope, using the $\times 20$ objective. D, the percentage of cells with neurites were calculated from 5 random fields in one well. The data were expressed as a mean percentage from 4 wells in one representative experiment. Statistical analyses were performed. The data are expressed as means \pm S.E. ${ }^{*} P<0.01$ compared with untreated cells. E, the effects of PD98059 on MAPK activation. Cells were incubated in $1 \%$ FBS medium under the indicated conditions, in the absence or the presence of PD98059. The cell lysates were analyzed via immunoblotting with anti-phospho ERK1/2 antibodies. Protein loading was normalized via immunoblotting with anti-ERK1/2 antibodies. 


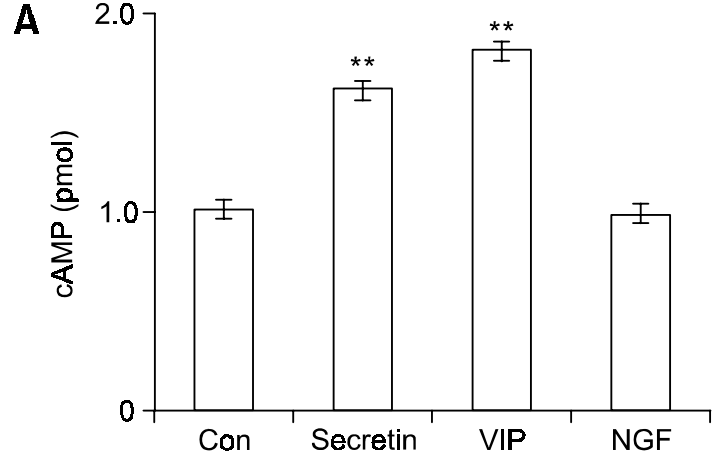

B

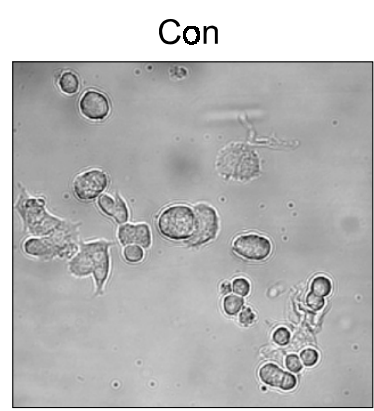

C

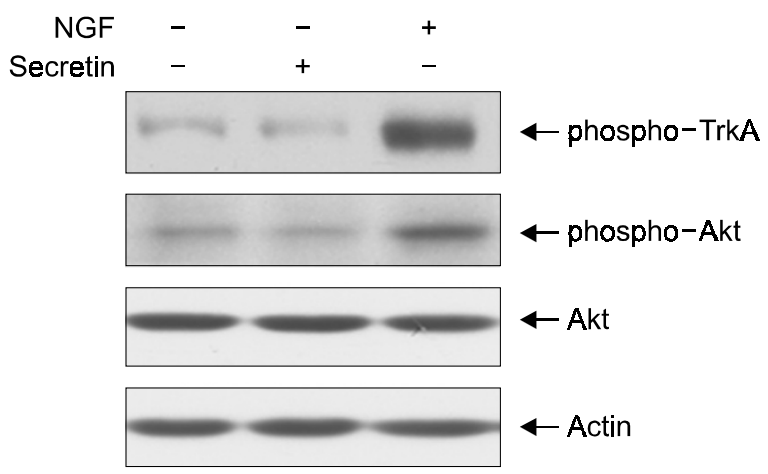

Figure 3. Secretin stimulates CAMP secretion in PC12 cells. A, the effects of secretin or NGF on the generation of cAMP. PC12 cells were stimulated with secretin $(1 \mu \mathrm{M})$, NGF $(10 \mathrm{ng} / \mathrm{ml})$, and VIP $(1 \mu \mathrm{M})$. The amounts of secreted cAMP in the supernatant were then measured. Statistically significant $\left.{ }^{* *} P<0.01\right)$ differences were observed in the VIP and secretin-treated cells, as compared with the untreated cells. B, the effects of H89 (1 $\left.\mu \mathrm{M}\right)$ on secretin-mediated neurite outgrowth. PC12 cells were plated on collagen-coated dishes. After 24 hours, the cells were then incubated in $1 \%$ FBS medium supplemented with secretin, in the presence of $\mathrm{H} 89(1 \mu \mathrm{M})$ or rapamycin. After 2 days, the cells were photographed with a confocal microscope using the $\times 20$ objective. C, effects of secretin or NGF on TrkA phosphorylation. PC12 cells were stimulated with either secretin or NGF. The cell lysates were analyzed via immunoblotting with anti-phospho TrkA/Akt antibodies. Blotting with anti-actin antibody was conducted to normalize the quantity of protein loading.

the generation of CAMP, as this was known to perform a critical function in neurite outgrowth in neurons (Rydel and Greene, 1988; Piiper et al., 2002). Levels of CAMP were shown to have increased upon stimulation with secretin, but this did not occur after NGF treatment (Figure 3A). Vasoactive intestinal peptide (VIP) $(1 \mu \mathrm{M})$ was known to stimulate the generation of CAMP, and was used as a positive control in this experiment (Iwasaki et al., 2001). To confirm this association with cAMP, we then pre-treated the cells with $\mathrm{H} 89(1 \mu \mathrm{M})$, a specific PKA inhibitor, direct downstream of cAMP, prior to the administration of secretin treatment, and then attempted to characterize the differentiation occurring in the PC12 cells. Secretin-mediated neurite outgrowth was blocked completely when the cells were pre-treated with $\mathrm{H} 89$ (Figure 3B). To show the differential mechanisms between secretin and NGF, we attempted to ascertain whether or not secretin activated the NGF receptor, TrkA. TrkA was phosphorylated upon stimulation with NGF, but not by secretin treatment (Figure $3 \mathrm{C}$ ). Phosphorylation of Akt, a downstream of TrkA, was also increased by the treatment with NGF, but not by secretin. These findings show that secretin may exert a neuritogenic effect via cAMP generation, rather than via the phosphorylation of Trk A.

\section{cAMP plays a critical role in the secretin-induced neuritogenic effect}

To further confirm the role of CAMP in secretinmediated neuritogenesis, we pre-treated the cells with $\mathrm{H} 89$ prior to administering secretin treatment, and then assessed the degree to which ERK1/2 phosphorylation had occurred. Phosphorylation was determined to have increased in a dose-dependent manner with the treatment of dbcAMP $(1 \mathrm{mM}), a$ cAMP analogue (Figure 4A), and this activation was inhibited by treatment with H89 (Figure 4B). Finally, to confirm the role of MAPK in CAMP-mediated differentiation, we pre-treated the cells with PD98059 
A

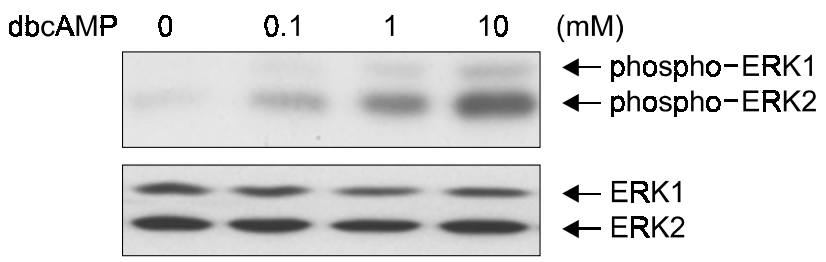

B

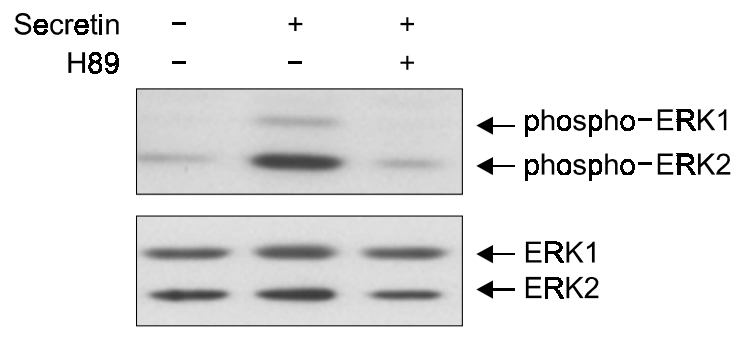

C

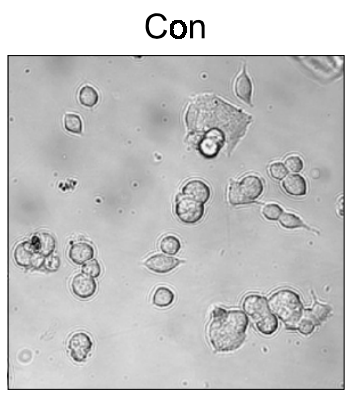

dbcAMP

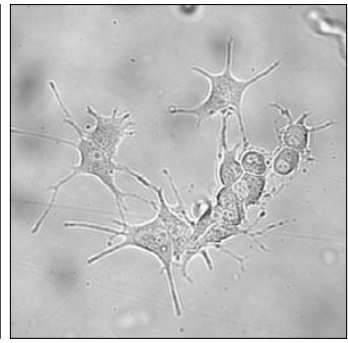

dbcAMP/

PD98059

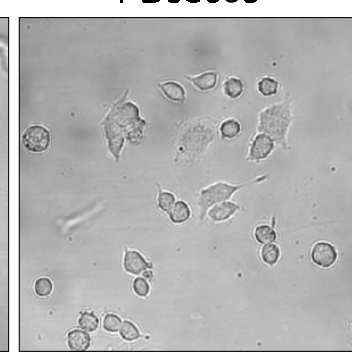

Figure 4. cAMP plays a critical role in the secretin-induced neuritogenic effect. A, effects of dbcAMP on the activity of MAPK. PC12 cells were stimulated for 10 minutes with the indicated concentrations of dbcAMP. The cell lysates were then analyzed via immunoblotting with anti-phospho ERK1/2 antibodies. Protein loading was normalized via immunoblotting with anti-ERK1/2 antibodies. B, the effects of $\mathrm{H} 89$ on the secretin-mediated activation of MAPK. The PC12 cells were stimulated with secretin $(1 \mu \mathrm{M})$, in the presence or absence of H89 $(1 \mu \mathrm{M})$. The cell lysates were analyzed via immunoblotting with anti-phospho ERK1/2 antibodies. C, the effects of PD98059 on dbcAMP-mediated neurite outgrowth. The PC12 cells were plated on collagen-coated dishes. After 24 hours, the cells were incubated in 1\% FBS medium supplemented with dbcAMP (1 mM), in the absence or presence of PD98059 $(2 \mu \mathrm{M})$, respectively. After 2 days, the cells were photographed with a confocal microscope, using the $\times 20$ objective.

prior to the administration of dbcAMP, and then observed the morphological changes which occurred in the cells. cAMP-mediated neurite outgrowth was suppressed dramatically as the result of PD98059 pre-treatment (Figure 4C). Overall, our findings implied that the CAMP-MAPK pathway might be critically involved in secretin-induced neuritogenesis.

\section{Discussion}

In our attempt to obtain a greater insight into the signal pathways involved in neuritogenesis, we have presented data which clearly suggest that the CAMP-MAPK pathway is involved in secretin-mediated neurite outgrowth. We further reveal that the PC12 cells express secretin receptors at the mRNA level.

The intracisternal injection of secretin altered dopamine metabolism and prolactin secretion in rats (Fuxe et al., 1979) and its influence on cAMP levels was reported in brain cells (Propst et al., 1979). Secretin has also been determined to be expressed in distinct neuronal populations within the cerebellum and cerebral cortex (Couvineau et al., 1986; Yung et al., 2001). It has also been reported that VIP, which is structurally similar to secretin, exhibited neurite-promoting effects in ventral spinal cord cells (Iwasaki et al., 2001). In the present study, we have determined that secretin induces neurite outgrowth in PC12 cells, supporting the notion that secretin plays a role as a neurotrophic factor. This can be explained by the remarkable homology in structure observed between VIP and secretin (Mutt et al., 1970). Amino acid residues consisted of 13 for VIP and 11 for secretin. Both peptides possessed $\mathrm{COOH}$ terminal amino acids; asparagines in VIP, and valine in secretin (Iwasaki et al., 2001). Although each receptor has a preferential affinity for its natural ligand, it may interact with a low affinity with the other peptide. Also, VIP and secretin cannot be shortened without a marked loss in biological potency. Thus, the C-terminal domains of VIP and secretin are necessary for effector activation. Therefore, it appears conceivable that amino acid residues of $\mathrm{COOH}$ terminal substances might play a role in neurite outgrowth.

In this study, we also observed that secretin activated the MAPK pathway, thereby indicating that secretin may elicit neuritogenesis, via the MAPK pathway. The involvement of the MAPK pathway in the commitment to cellular differentiation has been 
studied rather extensively (Tojima et al., 2003). The differential response between NGF and EGF has been attributed to the duration of MAPK activation; NGF induces a sustained activation of MAPK, but EGF treatment results in only a short-lived activation (Yasui et al., 2001). Here, we have demonstrated that secretin also induces sustained MAPK activation. The data collected in this study are in basic agreement with a previous finding that the sustained activation of MAPK exerted a marked influence on neuronal differentiation in the PC12 cells. However, the mechanism by which secretin or VIP exhibits trophic effects remains less than completely understood. These peptides may exert direct effects on neurons, or may act indirectly through other cells, including glial cells. Glial cells are known to secrete neurotrophic factors. In the case of VIP, the substance has been demonstrated to exhibit trophic properties, and appears to act directly via cAMP (Brenneman et al., 1985; Brenneman and Eiden, 1986). As was shown in this study, the secretin receptor was expressed in the PC12 cells. Therefore, secretin may act directly on this cell, and thus generate cAMP.

In conclusion, we have determined and characterized the effect of secretin on the differentiation of PC12 cells. Here, we have clearly shown that secretin induces neuritogenesis, and exerts its effects in a distinct manner, rather than via the activation of the NGF receptor, TrkA. Further experiments will be required in order to determine the manner in which the secretin signal is interconnected with the intracellular signal pathways for differentiation.

\section{Acknowledgment}

This study was supported by the National R \& D Program for Fusion Strategy of Advanced Technologies of Ministry of Science \& Technology (MOST).

\section{References}

Bouron A, Becker C, Porzig H. Functional expression of voltage-gated $\mathrm{Na}^{+}$and $\mathrm{Ca}^{2+}$ channels during neuronal differentiation of $\mathrm{PC} 12$ cells with nerve growth factor or forskolin. Naunyn Schmiedebergs Arch Pharmacol 1999; 359:370-77

Brenneman DE, Eiden LE, Siegel RE. Neurotrophic actions of VIP on spinal cord cultures. Peptide 1985;6:35-9

Brenneman DE, Eiden LE. VIP and electrical activity influence neuronal survival. Proc Natl Acad Sci USA 1986;83:1159-62

Caceres A, Kosik KS. Inhibition of neurite polarity by tau anti-sence oligonucleotide in primary cerebellar neurons. Nature 1990;343:461-3
Couvineau A, Gammeltoft S, Laburthe M. Molecular characteristics and peptide specificity of vasoactive intestinal peptide receptors from rat cerebral cortex. J Neurochem 1986;47:1469-75

Dong M, Miller LJ. Molecular pharmacology of the secretin receptor. Receptors Channels 2002;8:189-200

Esmaeli-Azad B, Mccarty JH, Feinstein SC. Sense and antisense transfection analysis of tau-function: tau influences net microtubule assembly, neurite outgrowth and neurite stability. J Cell Sci 1994;107:869-79

Fremeau RT Jr, Jensen RT, Charlton CG, Miller RL, O'Donohue TL, Moody TW. Secretin: specific binding to rat brain membranes. J Neurosci 1983;3:1620-5

Fuxe K, K Andersson, T Hokfelt, V Mutt, L Ferland, LF Agnati, D Ganten, S Said, P Eneroth, JA Gustafsson. Localization and possible function of peptidergic neurons and their interactions with central catecholamine neurons and the central action of gut hormones. Fed Proc 1979; $38: 2333-40$

Grumolato L, Louiset E, Alexandre D, Ait-Ali D, Turquier V, Fournier A, Fasolo A, Vaudry H, Anouar Y. PACAP and NGF regulate common and distinct traits of the sympatho adrenal lineage: effects on electrical properties, gene markers and transcription factors in differentiating PC12 cells. Eur J Neurosci 2003;17:71-82

Hagag N, Halegoua S, Viola M. Inhibition of growth factorinduced differentiation of PC12 cells by microinjection of antibody to ras p21. Nature 1986;319:680-2

Hirokawa N, Funakoshi T, Satoharada R, Kanai Y. Selective stabilization of tau in axons and microtubule-associated protein 2c in cell-bodies and dendrites contributes to polarized localization of cytoskeletal proteins in mature neurons. J Cell Biol 1996;132:667-79

Iwasaki Y, K Ikeda, Y Ichikawa, O Igarashi. Vasoactive intestinal peptide influences neurite outgrowth in cultured rat spinal cord neurons. Neurochem Res 2001;23:851-4

Leoni C, Menegon A, Benfenati F, Toniolo D, Pennuto M, Valtorta F. Neurite extension occurs in the absence of regulated exocytosis in PC12 subclones. Mol Biol Cell 1999;10:2919-31

Montminy M. Transcriptional regulation by cyclic AMP. Annu Rev Biochem 1997;66: 807-22

Mutt V, JE Jorpes, S Magnuson. Structure of porcine secretin. Eur J Biochem 1970;15:513-9

Nguyen TT, Scimeca JC, Filloux C, Peraldi P, Carpentire JL, Van Obberghen E. Co-regulation of the mitogen-activated protein kinase, extracellular signal-regulated kinase 1, and the $90-\mathrm{kDa}$ ribosomal S6 kinase in PC12 cells. Distinct effects of the neurotrophic factor, nerve growth factor, and the mitogenic factor, epidermal growth factor. $\mathrm{J}$ Biol Chem 1993;268:9803-10

Nozaki S, Nakata R, Mizuma H, Nishimura N, Watanabe $\mathrm{Y}$, Kohashi R, Watanabe $\mathrm{Y}$. In vitro auto-radiographic localization of (125)l-secretin receptor binding sites in rat brain. Biochem Biophys Res Commun 2002;292:133-7 
Pang L, Sawada T, Decker SJ, Saltiel AR. Inhibition of MAP kinase kinase blocks the differentiation of PC-12 cells induced by nerve growth factor. J Biol Chem 1995; 270:13585-8

Piiper A, Dikic I, Lutz MP, Leser J, Kronenberger B, Elez R, Cramer H, Muller-Esterl W, Zeuzem S. Cyclic AMP induces transactivation of the receptors for epidermal growth factor and nerve growth factor, thereby modulating activation of MAP kinase, Akt, and neurite outgrowth in PC12 cells. J Biol Chem 2002;277:43623-30

Propst F, L Moroder, E wunsch, B Hamprecht. The influence of secretin, glucagons and other peptides, of amino acids, prostaglandin endoperoxide analogues and diazepam on the level of adenosine 3',5'-cyclic monophosphate in neuroblastoma $\times$ glioma hybrid cells. J Neurochem 1979;32: 1495-500

Rydel RE, Greene LA. Cyclic AMP analogs promote survival and neurite outgrowth in cultures of rat sympathetic and sensory neurons independently of nerve growth factor. Proc Natl Acad Sci USA 1988;85:1257-61
Tojima T, Kobayashi S, Ito E. Dual role of cyclic AMPdependent protein kinase in neuritogenesis and synaptogenesis during neuronal differentiation. J Neurosci Res 2003;74:829-37

Traverse S, Gomez N, Paterson H, Marshall C, Cohen P. Sustained activation of the mitogen-activated protein (MAP) kinase cascade may be required for differentiation of PC12 cells. Comparison of the effects of nerve growth factor and epidermal growth factor. Biochem J 1992;288:351-55

Yasui H, Katoh H, Yamaguchi Y, Aoki J, Fujita H, Mori K, Negishi M. Differential responses to nerve growth factor and epidermal growth factor in neurite outgrowth of PC12 cells are determined by Rac1 activation systems. J Biol Chemistry 2001;276:15298-305

Yung WH, Leung PS, Ng SS, Zhang J, Chan SC, Chow BK. Secretin facilitates GABA transmission in the cerebellum. J Neurosci 2001;21:7063-8 